Technical Note

\title{
Reactor Laboratory for Biomedical Research in National Centre for Nuclear Research in Poland
}

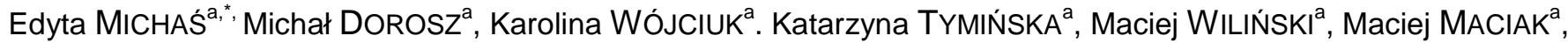 \\ Szymon DOMAŃSKI ${ }^{a}$, Grzegorz WOJTANIA ${ }^{a}$. Łukasz BARTOSIK ${ }^{a}$, Adam MAŁKIEWICZa ${ }^{a}$, Jan LECHNIAK ${ }^{a}$, Michał \\ Aleksander GRYZIŃSKI ${ }^{\mathrm{a}}$ \\ ${ }^{a}$ National Centre for Nuclear Research, Andrzeja Soltana 7, 05-400 Otwock, Poland \\ *E-mail address: edyta.michas@ncbj.gov.pl
}

\begin{abstract}
National Centre for Nuclear Research, NCBJ is one of the biggest research institutes in Poland, in which scientists deal with basic research in the various fields of subatomic physics, development of nuclear technologies and practical applications of nuclear physics methods, including those for nuclear medicine and radiotherapy. NCBJ operates the only Polish nuclear research reactor MARIA, around which a Reactor Laboratory for Biomedical Research, RLBR has been built in the last 4 years.

One of the main aims of the RLBR team is to adapt the H2 channel, one of the eight MARIA's horizontal channels, to a specific irradiation facility delivering a high flux thermal/epithermal neutron beam. The beam derived from the channel will be a tool for biological, physical and material studies for Boron Neutron Capture Therapy, BNCT.

While NCBJ is focused on building a neutron research facility, the Polish scientific community expressed its interest in BNCT development and implementation as an alternative therapy for cancer treatment. Through the working group meetings organized in the form of regular scientific workshops since 2015, it led to the establishment of a national scientific consortium dedicated to BNCT.

Polish Consortium for Boron Neutron Capture Therapy agreement was initially signed by twelve institutions including scientific institutes, universities and oncological centres in October 2019. National Centre for Nuclear Research was appointed the leader of the consortium. A year later the consortium was enlarged by two more institutions.
\end{abstract}

Key words: BNCT; radiobiology; radiochemistry; neutron beam; research reactor.

\section{Introduction}

Boron Neutron Capture Therapy (BNCT) is a two modality therapy enabling the treatment of head and neck cancers, brain cancers and melanoma. At the first stage of therapy, the patient receives a boron compound by intravenous infusion. The carrier attached to the boron compound is designed to selectively reach the cancer cells. In clinical practise, two boron carriers 4-borono-l-phenylalanine (BPA) and disodium mercaptoundecahydro-closo-dodecaborate (BSH) are used. A few hours after injection, the patient is irradiated by an epithermal neutron beam. The therapeutic effect is achieved through the nuclear capture and fission reaction taking place at the cellular level. The product of neutron irradiation of the stable isotope boron-10, boron-11, decays into alpha and lithium-7 particles. Currently, the therapy is being developed worldwide, using available reactor neutron sources and strongly developing accelerators.

\section{Research stand at $\mathrm{H} 2$ horizontal channel}

In the National Centre for Nuclear Research, in MARIA reactor the research facility on the $\mathrm{H} 2$ channel, is being constructed. $\mathrm{H} 2$ channel is one of the reactor horizontal channels. The uniqueness of the emerging position is influenced by the fact that it will be the only one in Poland, one of several in Europe enabling material and biological research. An additional strong point is a well-tied beam with a diameter of about $10 \mathrm{~cm}$. Another advantage of the constructed facility will be the possibility of switching between three various filter sets delivering a beam of, respectively, fast, epithermal or thermal neutrons. The estimated neutron flux will be about $10^{9} \mathrm{~cm}^{-2} \mathrm{~s}^{-1}$.

The emerging research infrastructure consists of 3 rooms: irradiation, preparation and monitoring. Samples will be transported through the special duct connecting the irradiation room with the preparation room. It will allow reducing the exposure of the researchers to radiation. In the corridor connecting both rooms, there will be apparatus controlling the 
experiment and the radiation conditions in the room. The first specification of the facility, in the current vision, has been presented in the publication of 2015 . $^{1}$ The above description is a significant update of the information posted at that time. Visualization of the facility and its most important elements:

- temporary shielding of the $\mathrm{H} 2$ horizontal channel (1),

- specialized irradiation table, prepared by the Nuclear Equipment Division, located in the NCBJ (2),

- crane for transporting samples between the irradiation room and the preparation room (3),

- duct connecting two rooms (4)

are shown in Figure 1.

The neutrons from the reactor core are in the thermal energy range. Obtaining an epithermal neutron beam requires the beam shaping system including the neutron converter, a subcritical system using the thermal neutron flux to initiate fission reactions in U-235. As a result of this reaction, fast neutrons will be emitted. The next element of the beam system is the intermediate channel - a cylindrical vessel placed inside the reactor pool between the converter and the stub pipe of the H2 channel.

The intermediate channel can be filled with nitrogen to make a gap in the pool water so the beam is formed and led into the $\mathrm{H} 2$ channel - a cylindrical hollow in the concrete wall of the pool. Inside the $\mathrm{H} 2$ channel, three various systems of moderators and filters will be installed to shift the neutron energy from fast to the epithermal or thermal range. The stand was designed for studies including beam and biological dosimetry, the creation of a BNCT planning system, as well as a system for monitoring the distribution of boron, both before and during irradiation.

The reactor will enable preclinical research as well as a training or preparation site for medical doctors and physicians working clinically. It will be a place where they become acquainted with the therapy, learn about the methods of dosimetry of mixed radiation fields, with the main contribution of the therapeutic neutron beam. The first recipient of the research facility will be the "Polish Consortium for Boron Neutron Capture Therapy". The competencies of Polish scientists focus on research on new boron carriers, which will increase the effectiveness of therapy. The consortium has also high competencies in the development of dosimetry methods.

\section{"Polish consortium for Boron Neutron Capture Therapy"}

The history of the "Polish consortium for Boron Neutron Capture Therapy" began in 2015. At that time, representatives of leading scientific institutions and universities from Poland met with specialists in the field of radiobiology, radiochemistry, physics, medical physics and medicine, dealing with the development of therapy in the world. It was the first BNCT Workshop in Poland. The second meeting took place in February 2016 in Wroclaw. During this meeting seven working

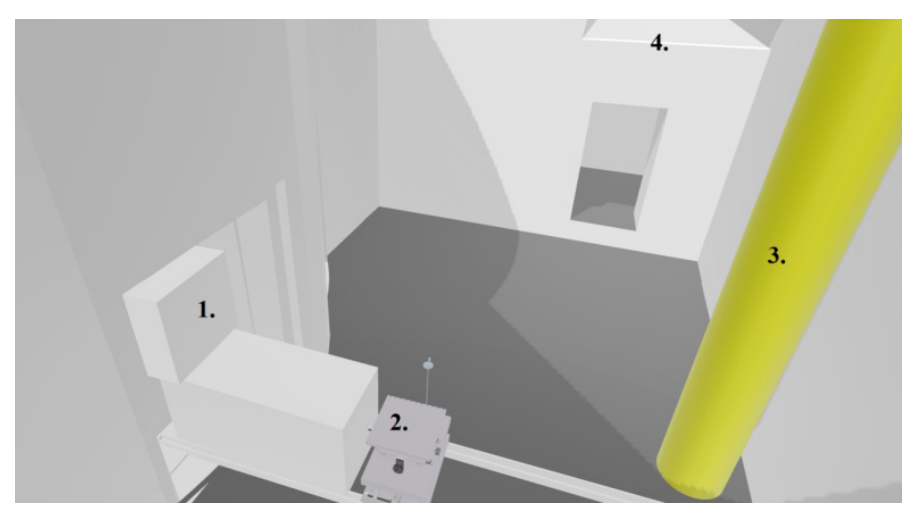

Figure 1. Visualization of the research stand at MARIA Research Reactor. The picture was made using a 3D design program. ${ }^{2}$

groups like boron biochemistry, research on biological material, preclinical studies, beam dosimetry, patient dosimetry, engineering and technical department, project promotion team were established. After the second Workshop, scientists have started work under a Letter of Intent, signed by institutions and universities from Poland and the world.

The next three editions of the workshops have led scientists from the medical community to join the BNCT-NeoBor scientific platform, treating BNCT as a new opening for oncology.

After several years of cooperation as Polish NeoBor - BNCT Scientific Platform, in October 2019, was signed the agreement to establish a "Polish Consortium for Boron Neutron Capture Therapy".

\section{Reactor Laboratory for Biomedical Research}

Interdisciplinary issues related to the constructed infrastructure have led to the emergence of the Reactor Laboratory for Biomedical Research, RLBR. The laboratory will enable research in the field of new neutron technologies, developmental studies on new dosimetry techniques including the design of detectors for measurements on mixed fields. In addition, the infrastructure being built will ensure the conduct of radiobiological, radiochemical, and also spectrometric and neutron activation analysis studies. Scientists working in the RLBR are divided into three main laboratories.

The main task of the Mixed Radiation Dosimetry Laboratory for RLBR is to assure and maintain the dosimetry system for the research stand. It will be achieved by three different systems of monitoring:

1. Radiation situation monitoring system in $\mathrm{H} 2$ irradiation room using two different probes connected to Reactor Dosimetric System) - Proportional counter based on argon/ $\mathrm{CO}_{2}$ for measurement of the ambient dose equivalent for gamma component and LB 6411 neutron probe for measurement of the ambient dose equivalent for neutrons.

2. Beam monitoring system to achieve proper beam monitoring system. It will be required to use a combination of a fission chamber with an ionization chamber or by placing a Geiger-Muller inside of the $\mathrm{H} 2$ channel. 
3. Dosimetric system at irradiation position - post-irradiation dosimetry will be achieved by passive detectors (TLDs and activation foils). Before irradiation combination of ionization chambers (equivalent to tissue and a nonhydrogen one).

As the research activity, the team for many years develops recombination chambers and appropriate recombination methods. It has experience in the dosimetry of mixed and difficult to characterize ionization fields and beam parameters, determining quantities such as absorbed dose of each radiation component or radiation quality factor. The team has original experience in measurements of the radiation field/beam for selected medical facilities: $\mathrm{x}$-ray radiotherapy, ${ }^{3,4} \mathrm{x}$-ray radiography, ${ }^{5,6}$ proton therapy, ${ }^{7,8}$ BNCT, ${ }^{9-11}$ and PET tomography. ${ }^{12}$

The activities of the Radiobiological Laboratory focus on exposure to radiation of human cell cultures. As a part of the laboratory's tasks, it is possible to study and determine the biological uptake mechanism of boron-containing molecules, as well as evaluating their effect on cell survival, apoptosis, signalling, and transport. Within carried out radiobiology research there was introduced the methods showing:

- DNA damage and efficiency of DNA repair (e.g. y-H2AX)

- cytogenetic effects (e.g. micronuclei assay)

- cells viability (e. g. clonogenic assay, MTS).

In the laboratory, it is possible to use a proprietary co-staining protocol developed to confirm $\gamma-\mathrm{H} 2 \mathrm{AX}$ foci as DNA-DSBs (double-strand breaks) using additionally 53BP1 immunofluorescent staining. Radiobiology Laboratory will introduce standard radiobiological methods for relative biological effectiveness (RBE) value counting based on the cell survival rate and DNA-DSBs.

There are plans to introduce new research models to BNCT. Besides research equalized on therapy, there are also conducted others, such as basic research at radiation (gamma, electrons, mixed neutron radiation) effects on human cells.

Moreover, it is planned to introduce Caenorhabditis elegans model for the application of thermal neutrons in BNCT and as a tool for in vivo application.

In 2019 the first experiments with new boron carriers were carried out. The synthesis of boron clusters conjugates with proteins or peptides, which have biological functions has been done. The constructs of boron conjugates are selectively captured by cancer cells using overexpression of the receptors of this protein. Evaluation of physicochemical properties of chemical compounds at every stage: lipophilicity studies, stability in buffers with different $\mathrm{pH}$ and ionic strength, as well as in cell culture media and physiological fluids. At this moment the scientists from the laboratory have basic competencies in design boron compounds for BNCT and their effects on cancer and healthy cells: cytotoxicity testing, cell death mechanisms and receptor affinity. The optimization of the concentration of boron atoms with a dose of neutrons to achieve the maximum toxic effect on cancer cells and at the same time reduce the effect on healthy cells has been done. Within the laboratory's activities, CBE (Compound Biological Effectiveness) determination for various types of cells has been performed. The Radiobiology Laboratory also deals with increasing radiosensitivity. In the future, the scientists intend to label boron carriers with isotopes for biodistribution testing in vivo and in vitro.

The aim of the Radiochemical Laboratory will be, among others:

- research on chemical dosimeters, development of existing methods and creation of new solutions,

- the creation of new methods for the purification of radioisotopes produced for medical, industrial and scientific purposes.

An increased interest in Instrumental Neutron Activation Analysis (INAA) using the Maria research reactor was also noted. Despite its years, this method is still interesting in many areas, including:

- testing of traces of heavy metals in various matrices,

- research on properly labelled radiopharmaceuticals,

- forensic science,

- material tests,

- determination of the level of metallic nanoparticles in cellular fractions.

- implementation of a Neutron Activation Analysis (NAA) for the determination of boron in biological samples for BNCT.

One of the directions of the Radiochemical Laboratory work was to minimize the dose received by the staff. For this purpose, INAA experiments were conducted on a number of commercially available polymers for 3D printers and containers for biological and chemical testing to identify possible radiological hazards. The results allowed us to optimize the process of biological and chemical experiments carried out so far.

Work is currently underway to create a new research stand to expand the research offer and to automate key elements of the sample measurement and analysis process. This will increase the repeatability of the method and increase the number of samples that can be measured, which is a challenge in the current stand configuration.

\section{Conclusions}

The process of preparing the research stand for BNCT on $\mathrm{H} 2$ horizontal channel was divided into 3 stages:

1. installation of the intermediate channel

2. preparation and installation of Beam Shaping Assembly system

3. preparation and installation of uranium converter.

The first stage of works has been completed in 2019, the second one is planned for 2021. After this work is completed, it will be possible to perform tests on an in vitro model. When 
the third stage of work will be done, the beam from the reactor core will reach clinical parameters.

The emerging research infrastructure in the reactor together with the laboratory facilities located next to it will enable "Polish Consortium for Boron Neutron Capture Therapy" to conduct world-level research in the field of boron carriers and new dosimetry equipment.

\section{Acknowledgements}

The authors would like to express gratitude to colleagues from Nuclear Facilities Operations Department in National Centre for Nuclear Research for their great work which has led to creation of this article.

This work was supported by internal funds within "Neutrony $\mathrm{H} 2$ " project.

\section{References}

1. Gryzinski MA, Maciak M, Wielgosz M. Summary of recent BNCT Polish programme and future plans. Appl Radiat Isot. 2015;106:10-17. https://doi.org/10.1016/j.apradiso.2015.07.047

2. Autodesk. Autodesk Inventor Professional. Autodesk; 2018.

3. Jakubowska E, Golnik N, Gryzinski M, Tulik P. Application of recombination chambers for determination of neutron ambient dose equivalent at the door to the maze of linear accelerator vault. Polish Journal of Medical Physics and Engineering. 2011;17(3):153158. https://doi.org/10.2478/v10013-011-0015-2

4. Tulik P, Tulik M, Maciak M, et al. Investigation of secondary mixed radiation field around a medical linear accelerator. Radiat Prot Dosimetry. 2018;180(1-4):252-255. doi:10.1093/rpd/ncx199

5. Golnik N, Mayer S, Zielczynski M. Recombination index of radiation quality of low-LET radiation. Nuclear Instruments and Methods in Physics Research Section B: Beam Interactions with Materials and Atoms. 2004;213:650-653. https://doi.org/10.1016/S0168-583X(03)01679-3

6. Tulik P, Lepak S, Domańska K, Jakubowska EA. Characterization of low-LET radiation fields for irradiation of biological samples using recombination chamber. In: Jabłoński R., Brezina T. (eds) Advanced Mechatronics Solutions. Advances in Intelligent Systems and Computing, vol 393. Springer, Cham. 2016. https://doi.org/10.1007/978-3-319-23923-1_20

7. Jakubowska E, Zielczynski M, Golnik N, Gryzinski MA, Krzeminski L. A ring-shaped recombination chamber for hadron therapy dosimetry. Radiat Prot Dosimetry. 2014;161(1-4):201-204. https://doi.org/10.1093/rpd/nct355

8. Jakubowska EA, Gryziński MA, Golnik N, et al. Ambient dose equivalent measurements in secondary radiation fields at proton therapy facility CCB IFJ PAN in Krakow using recombination chambers. Nukleonika. 2016;61(1):23-28. https://doi.org/10.1515/nuka-2016-0006

9. Tulik P, Golnik N, Zielczynski M. Recombination chambers filled with different gases-studies of possible application for BNCT beam dosimetry. Radiat Prot Dosimetry. 2004;110(1-4):669-673. https://doi.org/10.1093/rpd/nch160

10. Tulik P, Golnik N, Zielczynski M. Investigations of recombination chambers for BNCT beam dosimetry. Radiat Prot Dosimetry. 2007;126(1-4):648-651. https://doi.org/10.1093/rpd/ncm131

11. Tulik P, Golnik N. Studies of recombination chambers filled with nitrogen for BNCT dosimetry. Nukleonika. 2009;54(4):255-259.

12. Tulik P, Kowalska M, Golnik N, Budzynska A, Dziuk M. Measurements of the ionising radiation level at a nuclear medicine facility performing PET/CT examinations. Radiat Prot Dosimetry. 2017;174(4):501-509. https://doi.org/10.1093/rpd/ncw228 